\title{
Intrapleural combination therapy with bevacizumab and cisplatin for non-small cell lung cancer-mediated malignant pleural effusion
}

\author{
NAN DU ${ }^{1 *}$, XIAOSONG LI $^{1 *}$, FANG LI ${ }^{2}$, HUI ZHAO ${ }^{1}$, ZHONGYI FAN $^{1}$, \\ JUNXUN MA ${ }^{1}$, YAN FU ${ }^{1}$ and HUANRONG KANG ${ }^{1}$ \\ ${ }^{1}$ Department of Oncology, First Affiliated Hospital of Chinese PLA General Hospital, Beijing 100048; \\ ${ }^{2}$ Cancer Center, Chinese PLA General Hospital, Beijing 100853, P.R. China
}

Received October 21, 2012; Accepted January 3, 2013

DOI: 10.3892/or.2013.2349

\begin{abstract}
Malignant pleural effusion (MPE) is a common complication of advanced non-small cell lung cancer (NSCLC). Bevacizumab, a humanized monoclonal antibody against vascular endothelial growth factor (VEGF), has been shown to be efficient in suppressing the accumulation of pleural fluid. However, whether intrapleural delivery of bevacizumab can be used to treat MPE remains unknown. The aim of the present study was to evaluate the efficacy and safety of combined intrapleural therapy with bevacizumab and cisplatin, an antineoplastic agent, in controlling MPE. A total of 72 NSCLC study subjects with MPE were randomly assigned to one of two groups. The first group received intrapleural bevacizumab $(300 \mathrm{mg}$ ) with cisplatin $(30 \mathrm{mg}$ ) therapy and the second group received intrapleural cisplatin $(30 \mathrm{mg})$ therapy alone. Pleural fluid was collected from both groups prior to and following treatment. The levels of VEGF and carcinoembryonic antigen (CEA) in the pleural fluid were determined by ELISA. In 70 evaluable study subjects, the curative efficacy in the bevacizumab group was significantly higher than that found in the cisplatin group (83.33 vs. $50.00 \%$, respectively; $p<0.05$ ). Therapy with combined bevacizumab plus cisplatin significantly reduced VEGF levels in the pleural fluid $(\mathrm{p}<0.01)$. In the bevacizumab group, the levels of VEGF in the pleural fluid were significantly lower compared to those of the cisplatin group after treatment, which showed greater efficacy $(\mathrm{p}<0.01)$. In addition, combination therapy showed greater efficacy in the patients with high levels of VEGF expression ( $\mathrm{p}<0.01)$. There was no significant difference in grade III/IV adverse events between the two
\end{abstract}

Correspondence to: Dr Nan Du, Department of Oncology, The First Affiliated Hospital of Chinese PLA General Hospital, Beijing 100048, P.R. China

E-mail: dunan05@yahoo.com.cn

*Contributed equally

Key words: bevacizumab, non-small cell lung cancer, malignant pleural effusion, intrapleural chemotherapy groups. All procedures were well tolerated by the patients. Combined intrapleural therapy with bevacizumab and cisplatin was effective and safe in managing NSCLC-mediated MPE. We propose that VEGF expression levels in MPE could serve as a prognostic marker for bevacizumab therapy.

\section{Introduction}

Malignant pleural effusion (MPE) is a common complication of advanced metastatic non-small cell lung cancer (NSCLC). Abnormal fluid accumulation in the pleural space could lead to dyspnoea, cough and chest pain, which may eventually impair the normal function of the heart, and thus threaten patient survival (1). Since MPE presents with a high incidence and poor prognosis, characterized by a medium survival rate of 3 months, one of the highest priorities is to actively manage the pleural effusion in an attempt to improve the quality of life of the patients $(1,2)$. The current mode of therapy for patients with MPE primarily depends on local treatment, such as tube drainage, chemical pleurodesis and intrapleural administration of antineoplastic agents such as doxorubicin, cisplatin, cytarabine, carboplatin, etoposide, mitomycin C and 5-fluorouracil (3-6). Of these chemotherapeutic agents, cisplatin is the most commonly used for the treatment of NSCLC. It is thus the most frequently used drug for intrapleural therapy of pleural effusions caused by NSCLC (7). Although the current mode of therapy can occasionally alleviate the symptoms, the relapse rate is as high as $50 \%$. In addition, for most patients, no single treatment approach can achieve a satisfactory effect. Consequently, there is an urgent need for highly effective combinatorial therapies.

Vascular endothelial growth factor (VEGF) is an endothelial cell-specific growth factor that stimulates vasculogenesis and angiogenesis, and has been shown to be an essential intermediate in the formation of pleural effusions (8-10). Synthesis of VEGF by both autocrine and paracrine pathways (11) has shown it to be present in multiple solid cancers including NSCLC. Overexpression of VEGF in NSCLC can enhance its interaction with VEGF-specific receptors. Occupation of these receptors by VEGF could further stimulate the production of VEGF by both cancer and stroma cells, leading to local increases in the levels of VEGF in MPE. 
It is well documented that bevacizumab, a humanized monoclonal antibody raised against VEGF, is able to directly inhibit proliferation, migration and differentiation of vascular endothelial cells. Bevacizumab can also promote apoptosis of endothelial cells and suppress VEGF-induced neoangiogenesis and vascular permeability (12). Of note, bevacizumab has been shown to synergize with chemotherapeutic agents to block the accumulation of pleural fluid (13-17), thus making it a promising candidate for the clinical management of MPE. However, intravenous administration of bevacizumab requires a much higher dose to achieve a corresponding effect, whose side-effect might offset the ability of bevacizumab to diminish effusion (18). Furthermore, although intrapleural administration is a standard approach for managing pleural effusion, intrapleural administration of bevacizumab has not yet been reported for the treatment of MPE as a consequence of NSCLC. In the present study, we evaluated the efficacy and safety of local intrapleural administration of a combination of bevacizumab with cisplatin for the treatment of MPE. The main purpose of this study was to seek a more efficient therapeutic approach for the clinical management of these cancer-associated diseases.

\section{Materials and methods}

Research study subjects. This study was approved by the institutional review board of the First Affiliated Hospital of Chinese PLA Postgraduate Medical School (Beijing, China) and written informed consent was obtained from all participants (Approval no. ML25391). Seventy-two NSCLC subjects (excluding squamous cell carcinoma) with a presence of excess levels of pleural fluid were enrolled in this study from August 2009 to December 2011 inclusive. The research study subjects included 44 males and 28 females with a mean age of 52.50 years (range, 66-82 years). Primary tumors included adenocarcinoma in 48 cases, alveolar cell carcinoma in 17 cases and large cell carcinoma in seven cases. The study subjects were randomized into the bevacizumab group (intrapleural therapy of bevacizumab combined with cisplatin; $n=36$ ), and the cisplatin group (intrapleural cisplatin therapy alone; $n=36$ ). Two study subjects in the cisplatin group withdrew, including one study subject who was lost to follow-up after two cycles of chemotherapy, and one study subject who presented with dual cancer during treatment.

There was no statistically significant difference $(\mathrm{p}>0.05)$ between the two groups in terms of gender, age, clinical stage and pathological subtype (Table I). The MPE in the study subjects had been confirmed by imaging such as X-ray, CT scan and type-B ultrasonic scan in addition to cytological examination. The enrolled study subjects showed various symptoms including chest distress, cough and dyspnoea, at different levels.

The inclusion criteria included the following: a) advanced NSCLC confirmed histologically or pathologically, but not including squamous cancer; b) chest X-ray, ultrasonography or CT scan showing large areas of unilateral or bilateral plural effusion; c) malignant tumor cells found in the pleural fluid; d) no intrapleural injection of antineoplastic drugs or hardener within one month of recruitment to the study; e) Kamofsky score $($ KPS) $>60$, age $>18$ years at time of recruitment, and a predicted survival time $>3$ months; f) no major organ system dysfunction, and a blood cell count, as well as heart, liver and kidney test results within a normal range; g) previous chemotherapy to have been discontinued for $>6$ weeks prior to admission to this study.

The exclusion criteria included: a) history of allergy to biological agents; b) under current treatment with other antineoplastic agents; c) no detectable lesions; d) uncontrolled metastasis to the central nervous system; e) major organ dysfunction, such as congestive heart failure, malignant arrhythmia, angina requiring long-term medication, heart valve diseases, myocardial infarction and refractory hypertension; f) pregnant or breastfeeding women; g) infected wound; and h) history of refractory psychiatric diseases.

The criteria for withdrawal included: a) patients requesting withdrawal; b) stage III/IV adverse reactions related to bevacizumab therapy; c) poor study subject compliance; and d) disease progression. In addition, bevacizumab was discontinued if any of the following conditions occurred: a) wound dehiscence that needed intervention and associated complications; b) severe bleeding; c) severe arterial thrombus; d) hypertension crisis or hypertensive encephalopathy; e) reversible posterior leukoencephalopathy syndrome; and f) nephrotic syndrome.

Treatment protocol. After draining the pleural fluid by thoracentesis, study subjects were given intrapleural administration of either a combination of $30 \mathrm{mg}$ of cisplatin plus $300 \mathrm{mg}$ of bevacizumab or $30 \mathrm{mg}$ of cisplatin monotherapy. After $2 \mathrm{~h}$ of bed rest, study subjects were asked to turn over every $15 \mathrm{~min}$, in order to encourage full access of the delivered drugs to the chest wall. This treatment was given every two weeks. The tumor markers were examined at the end of the first treatment cycle. Detailed records of the responses following treatment were made by weekly blood monitoring and type-B ultrasonic tests. The end point of this study was the completion of 3 cycles of treatment. One week after the first intrapleural therapy, the enrolled NSCLC study subjects were given more than 3 cycles of conventional chemotherapy comprising paclitaxel $\left(175 \mathrm{mg} / \mathrm{m}^{2}\right)$ and carboplatin $(\mathrm{AUC}=6)$.

Evaluation of the standard of efficacy and safety. Recent objective responses were determined according to a previous study (19). Complete remission (CR) was considered when the accumulated fluid had disappeared and was stable for at least four weeks; partial remission (PR) was considered when $>50 \%$ of the accumulated fluid had disappeared, symptoms had improved, and the remaining fluid had failed to increase for at least four weeks; remission not obvious (NC) was considered when $<50 \%$ of the accumulated fluid had disappeared; progression (PD) was considered when the accumulated fluid had increased. The total efficiency was calculated by taking the sum of CR+PR. At the same time, the median progressionfree survival (PFS) and the median overall survival (OS) were assessed. Adverse reactions were evaluated by the Common Toxicity Evaluation Criteria (CTC) according to the National Cancer Institute (NCI). Qualify of Life (QOL) was evaluated by the Kanofsky score (KPS).

Quantitation of VEGF by enzyme-linked immunosorbent assay (ELISA). Pleural fluid was centrifuged at 4,000 rpm for 
Table I. Comparison of the clinical characteristics of the evaluable NSCLC study subjects of the two treatment groups.

\begin{tabular}{|c|c|c|c|}
\hline & Bevacizumab (\%) n=36 & Cisplatin $(\%) \mathrm{n}=34$ & P-value \\
\hline \multicolumn{4}{|l|}{ Age } \\
\hline$\geq 50$ & $20(55.56)$ & $18(52.9)$ & $>0.05$ \\
\hline $18-50$ & $16(44.44)$ & $16(46.7)$ & $>0.05$ \\
\hline \multicolumn{4}{|l|}{ Gender } \\
\hline Male & $19(52.8)$ & $19(55.6)$ & $>0.05$ \\
\hline Female & $17(47.2)$ & $15(444.1)$ & $>0.05$ \\
\hline \multicolumn{4}{|l|}{ Clinical stage } \\
\hline IV-M1 $\mathrm{a}^{\mathrm{a}}$ & $25(69.4)$ & 24 (70.6) & $>0.05$ \\
\hline IV-M1b & $11(30.6)$ & $10(29.4)$ & $>0.05$ \\
\hline \multicolumn{4}{|l|}{ Smoker } \\
\hline Ever smoker & $22(61.1)$ & $21(61.8)$ & $>0.05$ \\
\hline Never smoker & $14(38.2)$ & $13(38.2)$ & $>0.05$ \\
\hline \multicolumn{4}{|l|}{ EGFR mutation } \\
\hline Wild-type & $21(58.3)$ & $18(52.9)$ & $>0.05$ \\
\hline Mutand & $15(41.7)$ & $16(47.1)$ & $>0.05$ \\
\hline \multicolumn{4}{|l|}{ Subtype } \\
\hline Adenocarcinoma & $23(63.9)$ & $22(64.7)$ & $>0.05$ \\
\hline Alveolar cell carcinoma & $9(25.0)$ & $9(26.5)$ & $>0.05$ \\
\hline Large cell carcinoma & $4(11.1)$ & $3(8.8)$ & $>0.05$ \\
\hline \multicolumn{4}{|l|}{ Kamofsky score } \\
\hline$\geq 80$ & $27(75.0)$ & $25(73.5)$ & $>0.05$ \\
\hline$>60-<80^{a}$ & $9(25.0)$ & $9(26.5)$ & $>0.05$ \\
\hline \multicolumn{4}{|c|}{ Systematic treatment history (other regimen) } \\
\hline Yes & $25(69.4)$ & $23(67.6)$ & $>0.05$ \\
\hline No & $11(30.6)$ & $11(32.4)$ & $>0.05$ \\
\hline \multicolumn{4}{|l|}{ Local infusion history } \\
\hline Bleomycin monotherapy & $25(69.4)$ & $23(67.6)$ & $>0.05$ \\
\hline No & $11(30.6)$ & $11(32.4)$ & $>0.05$ \\
\hline
\end{tabular}

${ }^{\mathrm{a} K a r n o f s k y ~ s c o r e s ~ w e r e ~ d e c r e a s e d ~ b y ~ l a r g e ~ a m o u n t s ~ o f ~ p l e u r a l ~ f l u i d . ~}$

$10 \mathrm{~min}$ at $4^{\circ} \mathrm{C}$, following which the supernatant was collected and assessed by ELISA using the VEGF-A ELISA kit (USCN), according to the manufacturer's instructions. The assay plates were read using a Microplate Reader (Bio-Rad, model 550). Carcinoembryonic antigen (CEA) was determined by a chemiluminescence method.

Quantitative RT-PCR for VEGF-A. Cells $\left(\sim 10^{5}-10^{6}\right)$ were subjected to total RNA extraction using TRIzol reagent (Invitrogen Corp., Carlsbad, CA, USA). For RT-PCR, firststrand cDNA was synthesized from 0.2 to $1.0 \mu \mathrm{g}$ of total RNA with an oligo-dT primer (Gibco), according to the manufacturer's instructions. The sequences of the VEGF-A primers were: forward 5'-ctacctccaccatgccaagt-3', reverse 5'-aaatgctttc tccgetctga-3'.

Statistical analysis. Results are expressed as the means \pm standard error of the mean. Data were analyzed by the $\chi^{2}$ test and the rank sum test using SPSS 11.0 software. $\mathrm{p}<0.05$ was considered to indicate a statistically significant difference.
Table II. Comparison of the efficacy between the bevacizumab and cisplatin groups.

\begin{tabular}{lrrrrr}
\hline Group & $\mathrm{n}$ & $\mathrm{CR}$ & $\mathrm{PR}$ & $\mathrm{NC} / \mathrm{PD}$ & $\mathrm{OR}(\%)$ \\
\hline Cisplatin & 34 & 2 & 15 & 17 & 50.00 \\
Bevacizumab & 36 & 17 & 13 & 6 & 83.33 \\
\hline
\end{tabular}

$\mathrm{CR}$, complete remission; $\mathrm{PR}$, partial remission; $\mathrm{NC} / \mathrm{PD}$, remission not obvious/progression; OR, overall response.

\section{Results}

Short-term effect. The 70 evaluable enrolled study subjects had completed at least one cycle of treatment. The response rate for the combination therapy group was $83.33 \%$ (30/36 study subjects), while that for the cisplatin alone therapy group was $50.00 \%$ (17/34 study subjects). There was a statistically significant difference between the two groups in terms of 
Table III. Treatment cycles of intrapleural infusion in the bevacizumab and cisplatin groups.

\begin{tabular}{|c|c|c|c|c|c|}
\hline Group & Treatment cycle & Response (n) & $\mathrm{CR}$ & PR & Percentage $(\%)$ \\
\hline \multirow{3}{*}{ Bevacizumab $(n=36)$} & 2 & 13 & 5 & 8 & 36.11 \\
\hline & 3 & 15 & 11 & 4 & 41.67 \\
\hline & $\geq 4$ & 2 & 1 & 1 & 5.56 \\
\hline \multirow[t]{3}{*}{ Cisplatin $(n=34)$} & 2 & 2 & 0 & 2 & 5.88 \\
\hline & 3 & 4 & 0 & 4 & 11.77 \\
\hline & $\geq 4$ & 11 & 2 & 9 & 32.35 \\
\hline
\end{tabular}

CR, complete remission; PR, partial remission.

A

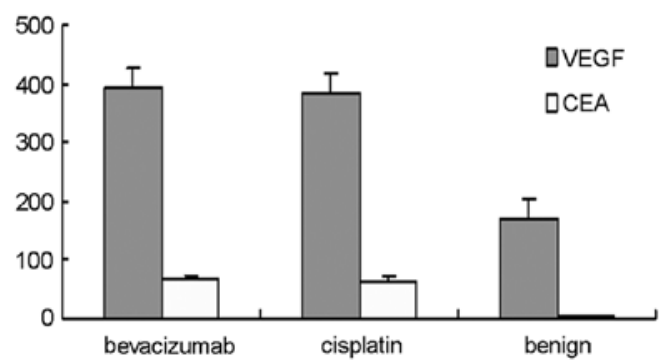

B

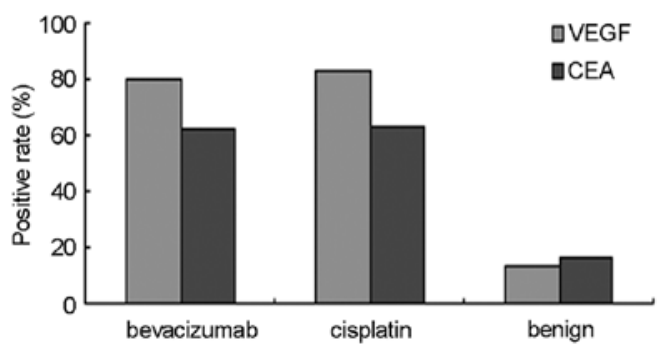

Figure 1. Comparison of progression-free survival (PFS) (A) and median overall survival (OS) (B) between the bevacizumab and cisplatin groups. (A) PFS: group 1, bevacizumab + cisplatin; group 2, cisplatin 5.3 vs. 4.5 months; $\mathrm{p}<0.05$; (B) OS: group 1, bevacizumab + cisplatin; group 2 , cisplatin 10.3 vs. 10.1 months; $\mathrm{p}>0.05$. VEGF, vascular endothelial growth factor; CEA, carcinoembryonic antigen.

stratification of MPE $(\mathrm{p}<0.05)$ (Table II). Following 2-3 cycles of treatment, the response rate was significantly increased in the bevacizumab group (Table III). However, the response rate only increased after 4 or more cycles of treatment in the cisplatin monotherapy group (Table III). Statistical analysis was not performed here due to the relatively small case numbers.

Long-term effect. The median PFS was found to be 5.3 months for the bevacizumab combination group and 4.5 months for the cisplatin alone group. This observation indicated that the addition of bevacizumab to the therapeutic regime could significantly extend the PFS of the study subjects presenting with MPE $(\mathrm{p}<0.05)$ (Fig. 1A). The median survival time was found to be 10.3 and 10.1 months, respectively, in the two groups ( $\mathrm{p}>0.05)$ (Fig. 1B).

Quality of life (QOL). Of the 36 study subjects in the combination therapy group, 30 study subjects were found to have an improved QOL (83.33\%), one study subject showed a stable QOL, and one study subject showed a reduced QOL. In the cisplatin monotherapy group, only $50.00 \%$ (15 cases) of the study subjects showed an improvement in their QOL.

Toxicity. There was no evidence of significantly increased toxicity following systemic chemotherapy in combination with intrapleural therapy. The major side-effects of bevacizumab treatment included bleeding and high blood pressure. Although the study subjects in both groups presented symptoms such as neutropenia, nausea, vomiting and diarrhea, all of the symptoms were typical side-effects of chemotherapy rather than being exclusively associated with bevacizumab treatment . Although one of the study subjects showed nasal mucous membrane bleeding, this observation did not affect follow-up treatment. The incidence of hypertension was also significantly higher in the combination therapy group $(\mathrm{p}<0.05)$. However, observation of hypertension in this group fell into stage I and II (although one case required oral antihypertensive therapy) and no subject withdrew from the study due to toxicity. No significant difference in grade III-IV adverse side-effects was seen between the two groups (Table IV).

Levels and positive rate of VEGF-A and CEA. The levels and positive rates of VEGF-A and CEA seen in the pleural effusion are shown in Fig. 2. The difference between the two groups of NSCLC study subjects treated with either cisplatin monotherapy or combined therapy with bevacizumab and cisplatin was not statistically significant $(p>0.05)$. The tumor marker CEA showed a significant difference between the study subjects presenting with MPE and those presenting with benign effusion $(p<0.01)$. Treatment with bevacizumab and cisplatin significantly decreased VEGF levels in the pleural fluid compared to that of the cisplatin monotherapy group before and after the respective treatments $(\mathrm{p}<0.01)$.

Relationship between VEGF expression and response rate. The VEGF positive status of the study subjects was deter- 
Table IV. Comparison of toxicity between the bevacizumab and cisplatin groups.

\begin{tabular}{|c|c|c|c|c|c|c|}
\hline \multirow[b]{2}{*}{ Group } & \multirow[b]{2}{*}{ Toxicity } & \multicolumn{5}{|c|}{ Grade } \\
\hline & & 0 & 1 & 2 & 3 & 4 \\
\hline \multirow[t]{7}{*}{ Bevacizumab $(n=36)$} & Leucocytopenia & 12 & 9 & 10 & 4 & 1 \\
\hline & Nausea and vomiting & 19 & 12 & 5 & 0 & 0 \\
\hline & Diarrhea & 30 & 4 & 2 & 0 & 0 \\
\hline & Rhinorrhagia & 34 & 2 & 0 & 0 & 0 \\
\hline & Hemoptysis or gastrointestinal bleeding & 36 & 0 & 0 & 0 & 0 \\
\hline & Hypertension & 27 & 7 & 2 & 0 & 0 \\
\hline & Proteinuria & 34 & 2 & 0 & 0 & 0 \\
\hline \multirow[t]{7}{*}{ Cisplatin $(n=34)$} & Leucocytopenia & 12 & 8 & 9 & 4 & 1 \\
\hline & Nausea and vomiting & 17 & 12 & 4 & 1 & 0 \\
\hline & Diarrhea & 28 & 3 & 2 & 1 & 0 \\
\hline & Rhinorrhagia & 34 & 0 & 0 & 0 & 0 \\
\hline & Hemoptysis or gastrointestinal bleeding & 34 & 0 & 0 & 0 & 0 \\
\hline & Hypertension & 32 & 2 & 0 & 0 & 0 \\
\hline & Proteinuria & 34 & 0 & 0 & 0 & 0 \\
\hline
\end{tabular}

A

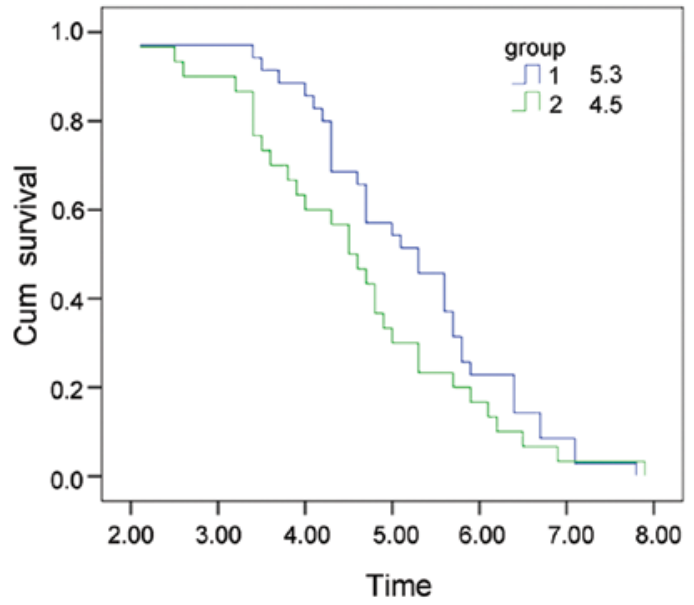

B

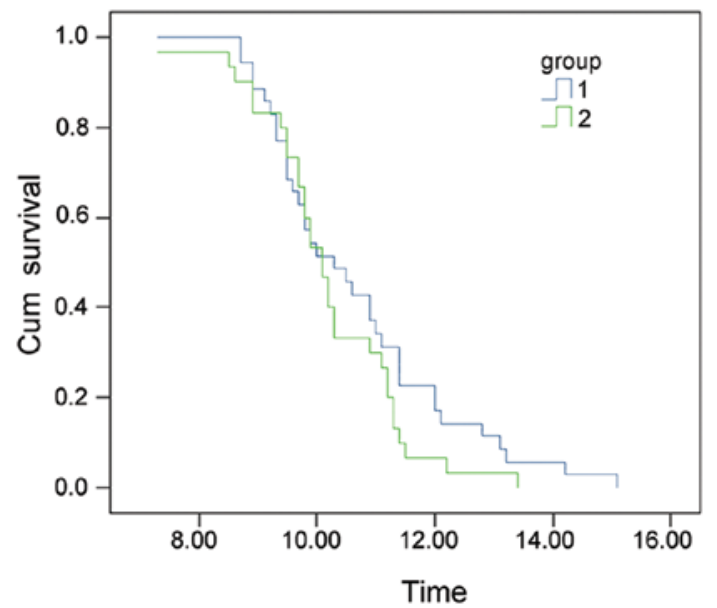

Figure 2. Levels of vascular endothelial growth factor (VEGF) (A) and carcinoembryonic antigen (CEA) (B) in the pleural fluid of NSCLC study subjects and benign pleural effusion subjects. The levels and positive rates of VEGF-A and CEA are shown. The difference between the two groups of NSCLC study subjects treated with either cisplatin alone or combined bevacizumab with cisplatin was not statistically significant ( $>0.05$ ). The tumor marker CEA showed a significant difference between the study subjects presenting with malignant pleural effusion and the subjects presenting with benign effusion ( $<<0.01$ ).

mined when the pleural VEGF was higher than the normal maximum value of $300.6 \mathrm{pg} / \mathrm{l}$. This was a value generated from a previous study which included 30 patients presenting with benign pleural effusions (unpublished data). The CEA-positive status was determined by comparison with the conventional standard value of pleural effusion $(5 \mathrm{ng} / \mathrm{l})$.

Bevacizumab was particularly efficient in the treatment of study subjects that were VEGF-positive $(\mathrm{p}<0.01)$ (Table V). Of the six VEGF negative study subjects, five showed no response to bevacizumab treatment, which suggested that bevacizumab therapy was specific to VEGF-positive patients. There were also five study subjects who were negative for CEA, but positive for VEGF yet showed an acceptable response to bevacizumab therapy (Table V). Of the 15 CEA-negative study
Table V. Relationship between efficacy and expression of VEGF and CEA in the bevacizumab group.

\begin{tabular}{lccccc}
\hline & \multicolumn{2}{c}{ VEGF $(\mathrm{n}=36)$} & & \multicolumn{2}{c}{$\operatorname{CEA}(\mathrm{n}=36)$} \\
\cline { 2 - 3 } \cline { 5 - 6 } & Positive & Negative & & Positive & Negative \\
\hline CR & 17 & 0 & & 12 & 5 \\
PR & 12 & 1 & & 8 & 5 \\
NC/PD & 1 & 5 & & 1 & 5
\end{tabular}

VEGF, vascular endothelial growth factor; CEA, carcinoembryonic antigen. CR, complete remission; PR, partial remission; NC/PD, remission not obvious/progression. 
A

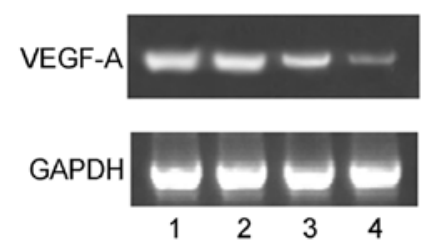

B

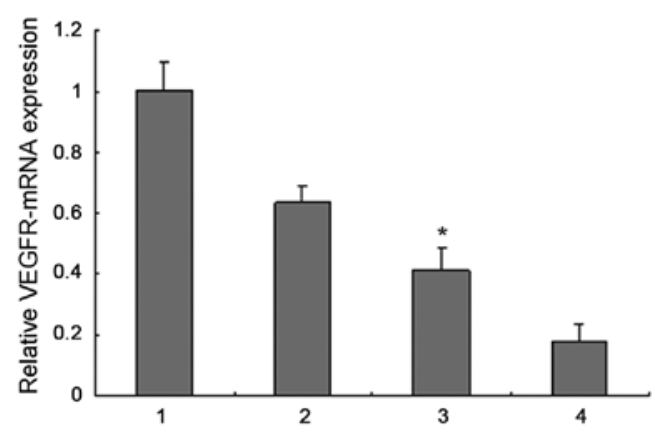

1 control 2 cisplatin 3 cisplatin plus bevacizumab 4 benign pleural effusion

Figure 3. VEGF-A mRNA expression levels in the pleural fluid of the various groups. (A and B) The mRNA expression of VEGF-A in the pleural effusion is shown. Compared to study subjects treated with cisplatin monotherapy, the pleural VEGF-A mRNA level was significantly lower than in study subjects treated with combination therapy $(\mathrm{p}<0.05)$ and as determined by real-time RT-PCR.

subjects, five were refractory to bevacizumab, while among the bevacizumab-refractory study subjects, five presented as CEA-positive, indicating that CEA was not a specific marker for bevacizumab treatment. Analysis of the 25 study subjects who were initially resistant to intrapleural therapy with bleomycin revealed that combination therapy with bevacizumab plus cisplatin provoked highly responsive regression of pleural effusion in 22 study subjects, an observation which may support an association between the VEGF-positive status and the actual response of study subjects to bevacizumab.

mRNA expression of VEGF-A in pleural effusion. The pleural VEGF-A mRNA was significantly lower in study subjects treated with combination therapy as compared with subjects treated with cisplatin alone, as determined by real-time RT-PCR (Fig. 3). The expression levels of VEGF-A mRNA (log copy number $/ \mu \mathrm{g}$ ) normalized to the internal control GAPDH in samples without therapy (before therapy), with cisplatin monotherapy and with combined cisplatin plus bevacizumab therapy were found to be $0.54 \pm 0.12,0.24 \pm 0.041$ and $0.15 \pm 0.029$, respectively. Expression of VEGF-A was significantly lower following treatment with either cisplatin monotherapy or with combined cisplatin plus bevacizumab $(p<0.05)$ (Fig. 4). There was also no difference in the basal expression of VEGF-A in both treatment groups $(\mathrm{p}=0.97)$ (Fig. 4). In addition, both treatment approaches were found to markedly decrease the mRNA expression levels of VEGF-A $(\mathrm{p}<0.01)$ in the study subjects. However, the extent to which VEGF-A mRNA decreased was much greater in subjects that had received the combination therapy compared to the subjects that had received cisplatin monotherapy $(\mathrm{p}<0.01)$. This observation suggested that bevacizumab could significantly reduce the levels of VEGF-A mRNA.

\section{Discussion}

Approximately 70-80\% of patients with NSCLC are diagnosed at relatively advanced stages (stage IIIB to IV) of their disease, with a 5-year survival rate for NSCLC of less than 5\% (1). Malignant pleural effusion (MPE) is seen in more than $50 \%$ of patients presenting with NSCLC $(1,2)$. The mortality rate of

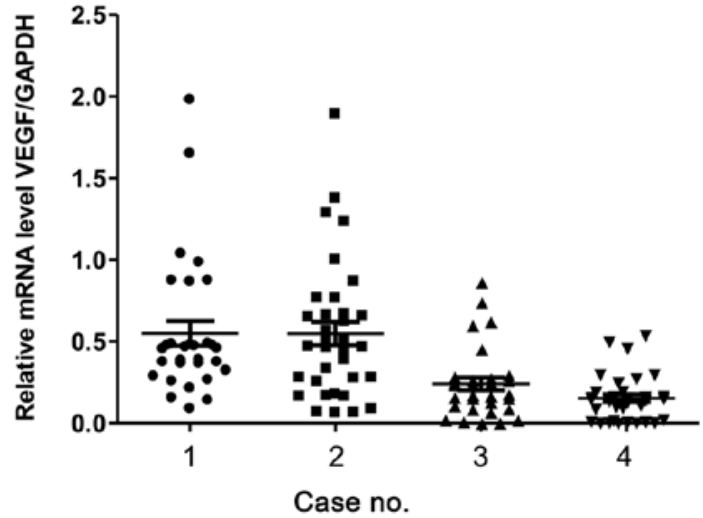

Figure 4. Ratiometric analysis of the VEGF-A mRNA/GAPDH levels in the pleural fluid of the various groups. VEGF-A expression was significantly lower following both treatments $(\mathrm{p}<0.05)$. Case 1 , cisplatin group before treatment; 2 , bevacizumab group before treatment; 3 , cisplatin group after treatment; 4, bevacizumab group after treatment. The results show that the VEGF-A mRNA/GAPDH levels were similar between the two groups before treatment $(\mathrm{p}=0.97)$. The VEGF-A mRNA/GAPDH levels in the bevacizumab group were significantly decreased as compared to the levels prior to treatment and compared to those of the cisplatin-treated group $(\mathrm{p}<0.01)$.

patients with MPE is very high (19). The detection of MPE can be a clinical indicator of local invasion or systemic metastasis of advanced lung cancer.

The current mode of therapy for patients with MPE involves primarily local treatment, such as tube drainage, chemical pleurodesis and intrapleural administration of antineoplastic agents, accompanied by the addition of a diuretic and albumin, and restricted intake of salt and water. Among these therapeutic approaches, intrapleural infusion is an important approach for managing pleural effusion. The drugs that are used intrapleurally include cisplatin, which can directly target tumor cells and stimulate chemical pleurisy to reduce the pleural effusion (20). However, as the ability of intrapleural chemotherapy to penetrate the tumor is restricted to only a few millimetres (21), the response rates are often disappointing, with an effectiveness of $0-66 \%$ (22). Furthermore, the clinical outcome of current modes of therapy is unstable and while they can occasionally alleviate the symptoms, there is no eventual benefit in patient survival. 
It has been demonstrated that in cancer patients the reabsorption of pleural fluid is blocked due to vascular and/or lymph node metastasis. By contrast, tumor cells can also secrete various growth factors which could lead to the local overexpression of VEGF, and thus contribute to elevated capillary permeability and fluid release from the capillary beds (10,23-27).

VEGF is a well-characterized angiogenic factor, with VEGF-A serving a prominent role in this regard, which on binding to its cognate receptors, primarily VEGFR-2, can increase vascular permeability, stimulate proliferation of vascular endothelial cells, promote the efflux of intravascular biological matrices, inhibit apoptosis of endothelial cells and activate enzymes that degrade the extracellular matrix. VEGF is thus able to facilitate the growth and extension of neovascularization. The ability of VEGF to promote vascular permeability renders it a key instigator of MPE $(28,29)$. The elevated efflux of plasma proteins provides the microenvironment a suitable matrix for the formation of pleural effusions (30-32).

Bevacizumab is a humanized monoclonal antibody against VEGF-A, which is able to specifically block the binding of VEGF-A to its receptor, and, by doing so, induces the degradation of existing tumor blood vessels and normalization of the remaining blood vessels (33). Bevacizumab can also substantially suppress the continuous growth and metastasis of tumor cells by repressing angiogenesis in the tumor tissues (34). Bevacizumab has been approved and recommended by the FDA in the treatment of certain types of metastatic cancer, including colon, breast and kidney tumors, as well as glioblastoma multiforme. The role of bevacizumab in suppressing malignant progression of pleural effusion has only recently been elucidated, and suggests that treatment with bevacizumab, either as monotherapy or in combination with other chemotherapeutic agents, could be useful in the treatment of malignant pleural effusion $(10,11)$. In addition, preclinical studies and clinical research, although still quite limited, have demonstrated that by inhibiting VEGF, treatment with bevacizumab may represent an effective way to prevent local fluid accumulation. To date, it has not been shown whether intrapleural bevacizumab therapy can be applied to MPE as a consequence of NSCLC.

On the basis of the aforementioned discussion, we compared the efficacy and safety of intrapleural combination therapy with bevacizumab and cisplatin with that of cisplatin monotherapy on MPE. Our results indicated that the intrapleural combination therapy of bevacizumab and cisplatin directed a very promising short-term effect, with a response rate of $85.71 \%$, a value which was significantly higher than that of cisplatin monotherapy (56.67\%).

Our results are consistent with a recent report that showed that pleural effusion could be markedly reduced by treatment with bevacizumab in combination with carboplatin and paclitaxel in a 63-year-old patient presenting with lung cancer (35). Similarly, intravenous or intraperitoneal administration of bevacizumab has been shown to be highly effective in the treatment of malignant ascites, at least in studies where only a limited number of study subjects were assessed (18,36-38).

We also showed that $88.57 \%$ of subjects presenting with both NSCLC and MPE overexpressed VEGF following treatment with bevacizumab. Additionally, study subjects that displayed originally high VEGF expression levels showed a more improved response than those subjects that were VEGF-negative.

The response rate for CEA-positive patients was found to be $57.1 \%$, where five subjects that were negative for CEA but positive for VEGF showed a satisfactory response to bevacizumab. By contrast, among the bevacizumab-resistant subjects, three were found to be CEA-positive, and this observation indicated that CEA was not a specific marker for bevacizumab treatment.

Analysis of the 25 subjects who were initially resistant to chemotherapy showed that treatment with a combination of bevacizumab plus cisplatin provoked regression of pleural effusion in 22 of the 25 subjects. This observation further supported the hypothesis that bevacizumab is highly effective in the subjects that were considered VEGF-positive. Furthermore, in most cases, the pleural effusion was well controlled after one or two treatment cycles, and the stratification of effusion was reduced following three cycles of treatment.

The median progression-free survival (PFS) for both treatment groups showed a significant difference, and indicated that the addition of bevacizumab to the therapeutic regimen may significantly extend the PFS of patients with MPE. Although the patients receiving bevacizumab treatment survived longer, the median survival (OS) for the two groups did not show any statistically significant difference. This could be due to the difference of the second-line treatment that some of the study subjects received.

Quantitative analysis of VEGF mRNA suggested that there was no difference in basal VEGF expression between subjects that had received any therapy. However, there was a significant difference between the subjects that had received combination therapy and the subjects that were treated with cisplatin monotherapy.

We also found that the VEGF mRNA levels in patients administered bevacizumab treatment were significantly lower than those found in subjects treated with cisplatin monotherapy. This observation suggested that bevacizumab may have downregulated VEGF-A gene expression, thereby inhibiting cell proliferation and migration, factors that are required for neoangiogenesis. This finding also suggested that bevacizumab may have suppressed the permeability of blood vessels, thereby eliminating the accumulation of pleural fluid. We suggest that this is likely the underlying mechanism by which bevacizumab inhibits the formation of MPE. Our results further confirmed the clinical significance of detecting VEGF mRNA and protein as biomarkers for the clinical management of MPE with bevacizumab.

Overexpression of VEGF in tumor tissues is associated with the metastatic potential of tumors and their rate of survival $(39,40)$. Thus, elevation in the serum levels of VEGF and overexpression of VEGF within the tumor tissue are considered to be associated with NSCLC staging and prognosis $(41,42)$. Of note, MPE that is caused by NSCLC also shows substantial increases in the levels of VEGF and its concentration exceeds that found in serum. By contrast, pleural effusion that is caused by lung infections, heart failure or cirrhosis of the liver does not appear to upregulate VEGF (43-48). Additionally, previous studies have shown that VEGF is the most highly overexpressed cytokine among 21 cytokines and chemokines that have been identified in both benign and malignant pleural effusion $(31,49,50)$. Markedly, 
the concentration of VEGF found in MPE can be considered an independent prognostic factor for tumor PFS and total survival. VEGF has been found to be associated with the sensitivity of tumors to chemotherapeutic agents. Therefore, treatment with an antibody specific to VEGF in patients with high levels of VEGF in the pleural effusion suggests that VEGF can be considered a biomarker for bevacizumab treatment of MPE $(18,51,52)$.

Our study provides evidence that combination therapy with intrapleural bevacizumab and cisplatin can efficiently treat MPE caused by NSCLC. This combination therapy can readily diminish effusion, with a positive health outcome for the patient. During this study, we did not detect any severe side-effects, indicating that this line of therapy is well tolerated. The combination therapy used in our study was able to control pleural effusion within a short period of time. Although mild hypertension was detected, it never exceeded grade II. We did not observe any proteinuria, thrombosis, gastrointestinal or pulmonary bleeding. Also, we did not have a single research study subject withdraw from the study due to toxicity. Although current evidence demonstrates that intrapleural bevacizumab does not lead to specific risks, we still recommend that some precautions be taken when managing patients with a significant tumor burden or in patients where squamous cell carcinoma is found in the main bronchus or in patients receiving surgery in the latter stages of the disease.

Treatment with intrapleural delivery of bevacizumab and cisplatin combination therapy is reliable, safe and feasible; it provides a novel approach for the management of MPE, and warrants further investigation. Our study demonstrated that the VEGF levels found in the pleural effusions of benign disease were markedly lower than those found in lung cancer. Moreover, VEGF expression can be significantly downregulated by intrapleural delivery of bevacizumab in lung cancer patients. This latter observation supports the theory that bevacizumab suppresses MPE by mechanisms dependent, in part, on the inhibition of VEGF expression.

We conclude that intrapleural combinatorial therapy with bevacizumab and cisplatin represents a novel treatment option for MPE. Intrapleural infusion does not necessarily impart an additional burden to the patients since it is also a routine therapeutic approach for pleural effusion. The preclinical and clinical study for intrapleural bevacizumab therapy has been successful and has also yielded some promising outcomes $(53,54)$. Our findings provide further evidence to an accumulating body of studies supporting the use of bevacizumab therapy, and suggest that intrapleural bevacizumab therapy could provide long-lasting effects in patients with MPE associated with advanced NSCLC. In addition, our investigation suggests that biomonitoring VEGF expression levels could be a reliable bioindicator of the clinical effectiveness of bevacizumab therapy.

\section{Acknowledgements}

This study was supported by grants from the National Health Research Foundation (no. W2010BX055), the National Natural Science Foundation of China (no. 81000994), the Chinese Wu Jie-ping Medical Foundation (no. 320.6750.1083), the Beijing Municipal Science and Technology Commission (no. Z121107001012080), and the National Natural Science Outstanding Youth Foundation of China (no. 39825111). The authors thank Dr Ye Qinong and Dr Sun Junzhong (Institute of Molecular Biology Medicine, Beijing, China), and Dr Ye Chuanzhong (Vanderbilt Epidemiology Center and Institute for Medical Center North, Nashville, TN, USA) for their helpful advice.

\section{References}

1. Sanz N, Aguado P, de Agustin JC, et al: Parapneumonic effussion. A review of 33 cases over 6 years. Cir Pediatr 18: 77-82, 2005 (In Spanish).

2. Ramalingam S and Belani C: Systemic chemotherapy for advanced non-small cell lung cancer: recent advances and future directions. Oncologist 13 (Suppl 1): 5-13, 2008.

3. Masuno T, Kishimoto S, Ogura T, et al: A comparative trial of LC9018 plus doxorubicin and doxorubicin alone for the treatment of malignant pleural effusion secondary to lung cancer. Cancer 68: 1495-1500, 1991.

4. Rusch VW, Figlin R, Godwin D and Piantadosi S: Intrapleural cisplatin and cytarabine in the management of malignant pleural effusions: a Lung Cancer Study Group trial. J Clin Oncol 9: 313-319, 1991.

5. Luh KT, Yang PC, Kuo SH, Chang DB, Yu CJ and Lee LN: Comparison of OK-432 and mitomycin $\mathrm{C}$ pleurodesis for malignant pleural effusion caused by lung cancer. A randomized trial. Cancer 69: 674-679, 1992.

6. Tohda Y, Iwanaga T, Takada M, et al: Intrapleural administration of cisplatin and etoposide to treat malignant pleural effusions in patients with non-small cell lung cancer. Chemotherapy 45: 197-204, 1999.

7. Ishida A, Miyazawa T, Miyazu Y, et al: Intrapleural cisplatin and OK432 therapy for malignant pleural effusion caused by non-small cell lung cancer. Respirology 11: 90-97, 2006.

8. Senger DR, Galli SJ, Dvorak AM, Perruzzi CA, Harvey VS and Dvorak HF: Tumor cells secrete a vascular permeability factor that promotes accumulation of ascites fluid. Science 219: 983-985, 1983.

9. Fontanini G, Faviana P, Lucchi M, et al: A high vascular count and overexpression of vascular endothelial growth factor are associated with unfavourable prognosis in operated small cell lung carcinoma. Br J Cancer 86: 558-563, 2002.

10. Sack U, Hoffmann M, Zhao XJ, et al: Vascular endothelial growth factor in pleural effusions of different origin. Eur Respir J 25: 600-604, 2005.

11. Kobold S, Hegewisch-Becker S, Oechsle K, Jordan K, Bokemeyer C and Atanackovic D: Intraperitoneal VEGF inhibition using bevacizumab: a potential approach for the symptomatic treatment of malignant ascites? Oncologist 14: 1242-1251, 2009.

12. Ferrara N, Gerber HP and LeCouter J: The biology of VEGF and its receptors. Nat Med 9: 669-676, 2003.

13. Pichelmayer O, Zielinski C and Raderer M: Response of a nonmalignant pleural effusion to bevacizumab. N Engl J Med 353: 740-741, 2005.

14. Neragi-Miandoab S: Malignant pleural effusion, current and evolving approaches for its diagnosis and management. Lung Cancer 54: 1-9, 2006.

15. Cohen MH, Gootenberg J, Keegan P and Pazdur R: FDA drug approval summary: bevacizumab (Avastin) plus Carboplatin and Paclitaxel as first-line treatment of advanced/metastatic recurrent nonsquamous non-small cell lung cancer. Oncologist 12: 713-718, 2007.

16. Ribeiro SC, Vargas FS, Antonangelo L, et al: Monoclonal antivascular endothelial grow th factor antibody reduces fluid volume in an experimental model of inflammatory pleural effusion. Respirology 14: 1188-1193, 2009.

17. Teixeira LR, Vargas FS, Acencio MM, et al: Blockage of vascular endothelial growth factor (VEGF) reduces experimental pleurodesis. Lung Cancer 74: 392-395, 2011.

18. Pichelmayer O, Gruenberger B, Zielinski C and Raderer M: Bevacizumab is active in malignant effusion. Ann Oncol 17: 1853, 2006.

19. Sahn SA: Management of malignant pleural effusions. Monaldi Arch Chest Dis 56: 394-399, 2001.

20. Musani AI: Treatment options for malignant pleural effusion. Curr Opin Pulm Med 15: 380-387, 2009. 
21. Los G, Mutsaers PH, van der Vijgh WJ, Baldew GS, de Graaf PW and McVie JG: Direct diffusion of cis-diamminedichloroplatinum(II) in intraperitoneal rat tumors after intraperitoneal chemotherapy: a comparison with systemic chemotherapy. Cancer Res 49: 3380-3384, 1989.

22. Walker-Renard PB, Vaughan LM and Sahn SA: Chemical pleurodesis for malignant pleural effusions. Ann Intern Med 120: 56-64, 1994.

23. Aslam $\mathrm{N}$ and Marino CR: Malignant ascites: new concepts in pathophysiology, diagnosis, and management. Arch Intern Med 161: 2733-2737, 2001.

24. Grove CS and Lee YC: Vascular endothelial growth factor: the key mediator in pleural effusion formation. Curr Opin Pulm Med 8: 294-301, 2002.

25. Patane S, Marte F, Di Bella G and Davi M: Pericardial effusion with elevated serum carbohydrate antigen 125 levels and ovarian tumor mass. Int J Cardiol 127: e105-e107, 2008.

26. Yokoyama T, Tanaka A, Kato S and Aizawa H: Multiple myeloma presenting initially with pleural effusion and a unique paraspinal tumor in the thorax. Intern Med 47: 1917-1920, 2008.

27. Chen Y, Liang B, Zhao YJ, Wang SC, Fan YB and Wu GP: Transcription expression and clinical significance of vascular endothelial growth factor mRNA and endostatin mRNA in pleural effusions of patients with lung cancer. Diagn Cytopathol 40: 287-291, 2012

28. Folkman J, Merler E, Abernathy C and Williams G: Isolation of a tumor factor responsible for angiogenesis. J Exp Med 133: 275-288, 1971.

29. Mohammed KA, Nasreen N, Hardwick J, Logie CS, Patterson CE and Antony VB: Bacterial induction of pleural mesothelial monolayer barrier dysfunction. Am J Physiol Lung Cell Mol Physiol 281: L119-L125, 2001.

30. Folkman J: Tumor angiogenesis and tissue factor. Nat Med 2 : 167-168, 1996

31. Zebrowski BK, Yano S, Liu W, et al: Vascular endothelial growth factor levels and induction of permeability in malignant pleural effusions. Clin Cancer Res 5: 3364-3368, 1999.

32. Strizzi L, Catalano A, Vianale G, et al: Vascular endothelia growth factor is an autocrine growth factor in human malignant mesothelioma. J Pathol 193: 468-475, 2001.

33. Presta LG, Chen H, O'Connor SJ, et al: Humanization of an anti-vascular endothelial growth factor monoclonal antibody for the therapy of solid tumors and other disorders. Cancer Res 57: 4593-4599, 1997.

34. Shaheen RM, Tseng WW, Vellagas R, et al: Effects of an antibody to vascular endothelial growth factor receptor- 2 on survival, tumor vascularity, and apoptosis in a murine model of colon carcinomatosis. Int J Oncol 18: 221-226, 2001.

35. Hama M, Komatsu Y and Hachiya T: A case of lung cancer showing marked reduction of pleural effusion by bevacizumab in combination with carboplatin and paclitaxel. Gan To Kagaku Ryoho 38: 1877-1879, 2011 (In Japanese).

36. Numnum TM, Rocconi RP, Whitworth J and Barnes MN: The use of bevacizumab to palliate symptomatic ascites in patients with refractory ovarian carcinoma. Gynecol Oncol 102: 425-428, 2006.

37. Hamilton CA, Maxwell GL, Chernofsky MR, Bernstein SA, Farley JH and Rose GS: Intraperitoneal bevacizumab for the palliation of malignant ascites in refractory ovarian cancer. Gynecol Oncol 111: 530-532, 2008.
38. Kesterson JP, Mhawech-Fauceglia P and Lele S: The use of bevacizumab in refractory ovarian granulosa-cell carcinoma with symptomatic relief of ascites: a case report. Gynecol Oncol 111: 527-529, 2008

39. Volm M, Koomagi R and Mattern J: Prognostic value of vascular endothelial growth factor and its receptor Flt-1 in squamous cell lung cancer. Int J Cancer 74: 64-68, 1997.

40. Kassim SK, El-Salahy EM, Fayed ST, et al: Vascular endothelial growth factor and interleukin- 8 are associated with poor prognosis in epithelial ovarian cancer patients. Clin Biochem 37: 363-369, 2004.

41. Kumar H, Heer K, Lee PW, et al: Preoperative serum vascular endothelial growth factor can predict stage in colorectal cancer. Clin Cancer Res 4: 1279-1285, 1998.

42. Matsuyama W, Hashiguchi T, Mizoguchi A, et al: Serum levels of vascular endothelial growth factor dependent on the stage progression of lung cancer. Chest 118: 948-951, 2000.

43. Cheng D, Rodriguez RM, Perkett EA, et al: Vascular endothelial growth factor in pleural fluid. Chest 116: 760-765, 1999.

44. Kraft A, Weindel K, Ochs A, et al: Vascular endothelial growth factor in the sera and effusions of patients with malignant and nonmalignant disease. Cancer 85: 178-187, 1999.

45. Thickett DR, Armstrong L and Millar AB: Vascular endothelial growth factor (VEGF) in inflammatory and malignant pleural effusions. Thorax 54: 707-710, 1999.

46. Yanagawa H, Takeuchi E, Suzuki Y, Ohmoto Y, Bando H and Sone S: Vascular endothelial growth factor in malignant pleural effusion associated with lung cancer. Cancer Immunol Immunother 48: 396-400, 1999.

47. Verheul HM, Hoekman K, Jorna AS, Smit EF and Pinedo HM: Targeting vascular endothelial growth factor blockade: ascites and pleural effusion formation. Oncologist 5 (Suppl 1): 45-50, 2000.

48. Ishimoto O, Saijo Y, Narumi K, et al: High level of vascular endothelial growth factor in hemorrhagic pleural effusion of cancer. Oncology 63: 70-75, 2002.

49. Kishiro I, Kato S, Fuse D, Yoshida T, Machida S and Kaneko N: Clinical significance of vascular endothelial growth factor in patients with primary lung cancer. Respirology 7: 93-98, 2002.

50. Momi H, Matsuyama W, Inoue K, et al: Vascular endothelial growth factor and proinflammatory cytokines in pleural effusions. Respir Med 96: 817-822, 2002.

51. Harlozinska A, Sedlaczek P, Kulpa J, et al: Vascular endothelial growth factor (VEGF) concentration in sera and tumor effusions from patients with ovarian carcinoma. Anticancer Res 24: 1149-1157, 2004.

52. Rudlowski C, Pickart AK, Fuhljahn C, et al: Prognostic significance of vascular endothelial growth factor expression in ovarian cancer patients: a long-term follow-up. Int J Gynecol Cancer 16 (Suppl 1): 183-189, 2006.

53. Watanabe M, Boyer JL and Crystal RG: AAVrh.10-mediated genetic delivery of bevacizumab to the pleura to provide local anti-VEGF to suppress growth of metastatic lung tumors. Gene Ther 17: 1042-1051, 2010.

54. Konner JA, Grabon DM, Gerst SR, et al: Phase II study of intraperitoneal paclitaxel plus cisplatin and intravenous paclitaxel plus bevacizumab as adjuvant treatment of optimal stage II/III epithelial ovarian cancer. J Clin Oncol 29: 4662-4668, 2011. 